\title{
REMARQUES SUR LES IDÉAUX DE POLYNÔMES ET DE FORMES DIFFÉRENTIELLES EXTÉRIEURES II
}

\author{
CLAUDIO PERELLI CIPPO \\ Università Cattolica del Sacro Cuore \\ Largo Gemelli 1, I-20123 Milano, Italia
}

1. Une construction analogue à celle de la première partie de cette conférence peut être appliquée aussi aux idéaux de formes extérieures à coefficients indéterminés (v. [2], [5]).

Considérons pour cela le $R$-module gradué $A=\oplus A^{l}: l=0, \ldots, n$ où $A^{l}$ est l'espace vectoriel des formes extérieures de degré $l$ en $n$ indéterminées (soient $\mathrm{d} x^{1}, \ldots, \mathrm{d} x^{n}$ ) à coefficients réels avec la multiplication extérieure: si nous voulons garder les notations de la première partie, la dimension de l'espace vectoriel $A^{l}$ des formes de degré $l$ sera $\left(\begin{array}{c}N+n \\ l\end{array}\right)$ (et non plus $\left(\begin{array}{c}n+l-1 \\ n\end{array}\right)$ ); le degré $l$ des formes que nous aurons a considérer ne pourra être plus grand que $n$ (parce que les formes de degré plus grand sont identiquement nulles) ou même plus grand que $n / 2$ (parce que, au delà, la dimension de leur espace va diminuer...).

Ainsi l'ensemble des idéaux de formes extérieures (idéaux dans le $R$-module $A$ ) peut être partitionné en classes

$$
\mathcal{H}^{\rho}\left(l_{1}(1), \ldots, l_{1}\left(m_{1}\right), l_{2}\left(m_{1}+1\right), \ldots, l_{2}\left(m_{2}\right), \ldots, l_{q}\left(m_{q}\right) ; n\right)
$$

ou, s'il n'y a pas de confusion à craindre, $\mathcal{H}^{\rho}\left(l_{1}, \ldots, l_{q} ; n\right)$, en fonction des indices $l_{1}, \ldots, l_{q}$, $m_{1}, \ldots, m_{q}$ et du multi-indice $\rho$. Chacune de cettes classes a une structure standard de variété, fibrée plusieures fois, avec une dimension finie.

Plus précisément: pour $q>1, \rho:=\left(r_{l_{1}+1}, \ldots, r_{l_{q}}\right) \in \mathbb{N}^{l_{q}-l_{1}}$, soit $\mathcal{H}^{\rho}\left(l_{1}, \ldots, l_{q} ; n\right)$, le sous-ensemble de $\mathcal{I}\left(l_{1}, \ldots, l_{q} ; n\right)$ (c'est à dire de l'ensemble des idéaux homogènes $I=\oplus I_{l}$ engendrés par $m_{1}$ formes extérieures de degré $l_{1}, m_{2}-m_{1}$ formes de degré $l_{2}, \ldots, m_{q}-$ $m_{q-1}$ de degré $l_{q}$, tels que les générateurs du même degré soient linéairement indépendants et que ceux de degré plus élevé soient algébriquement indépendants de ceux de degré inférieur), défini par la condition

$\left.\operatorname{III}_{\rho}\right)$ Le sous-espace vectoriel $E_{l}$ de $I_{l} \quad\left(l_{1}<l \leq l_{q}\right)$ (engendré, par combinaisons à coefficients formes d'ordre convenable, par les composantes $I_{h}$ de $I$ avec $h<l$ ) a

1991 Mathematics Subject Classification: Primary 58A15: Secondary 13A15.

Research supported by MURST $60 \%$.

The paper is in final form and no version of it will be published elsewhere. 
dimension: $\operatorname{dim} E_{l}=r_{l}$.

On a immédiatement

$$
\mathcal{I}\left(l_{1}, \ldots, l_{q} ; n\right)=\bigcup \mathcal{H}^{\rho}\left(l_{1}, \ldots, l_{q} ; n\right): \rho \in \mathbb{N}^{l_{q}-l_{1}}
$$

et, ayant posé $\mathcal{H}^{r}\left(l_{1}, \ldots, l_{q} ; n\right):=\bigcup \mathcal{H}^{\rho}\left(l_{1}, \ldots, l_{q} ; n\right):|\rho|=r$, on a aussi

$$
\mathcal{I}\left(l_{1}, \ldots, l_{q} ; n\right)=\bigcup H^{r}\left(l_{1}, \ldots, l_{q} ; n\right): r \in \mathbb{N} .
$$

Si toutes les valeurs $r$ qui figurent dans $\rho$ sont maxima, soit $\bar{r}=|\rho|$, nous écrivons plus simplement $\mathcal{H}\left(l_{1}, \ldots, l_{q} ; n\right)$ en lieu de $\mathcal{H}^{\rho}\left(l_{1}, \ldots, l_{q} ; n\right)$.

Posons encore: $\mathcal{K}^{0}\left(l_{1}, \ldots, l_{q} ; n\right):=\mathcal{I}\left(l_{1}, \ldots, l_{q} ; n\right) \backslash \mathcal{H}\left(l_{1}, \ldots, l_{q} ; n\right)$

$\mathcal{K}^{i}\left(l_{1}, \ldots, l_{q} ; n\right):=\mathcal{K}^{i-1}\left(l_{1}, \ldots, l_{q} ; n\right) \backslash \mathcal{H}^{\bar{r}-i}\left(l_{1}, \ldots, l_{q} ; n\right)$.

On peut alors démontrer la

Proposition 1. $\mathcal{H}\left(l_{1}, \ldots, l_{q} ; n\right)$ est ouvert (partout dense) dans $\mathcal{I}\left(l_{1}, \ldots, l_{q} ; n\right)$; $\mathcal{H}^{\bar{r}-1}\left(l_{1}, \ldots, l_{q} ; n\right)$ est ouvert dans $\mathcal{K}^{0}\left(l_{1}, \ldots, l_{q} ; n\right)$, et, en général $\mathcal{H}^{\bar{r}-i}\left(l_{1}, \ldots, l_{q} ; n\right)(i \geq$ 1) sont ouverts dans $\mathcal{K}^{i-1}\left(l_{1}, \ldots, l_{q} ; n\right)$.

2. Parmi les idéaux de formes extérieures, le cas le plus important que l'on rencontre est celui des idéaux différentiels, fermés par rapport à l'opération de la différentiation extérieure, déterminés par un système différentiel extérieur $\sum$, comme ceux qui sont le prolongement de équations ou de systèmes de équations aux dérivées partielles.

Dans ce cas il est bien connu, que l'ordinairété et la non ordinairété des éléments intégraux de $\sum$ sont détérminées par des entiers, les indices de Cartan, qui sont introduits par des raisonnements d'algèbre linéaire et qui expriment les rangs de matrices (les matrices des systèmes polaires) déduites de $\sum$.

Mais, pour effectuer le pas successif, il est convenable de introduire quelque notation nouvelle: en premier lieu nous ne traitons plus de éléments intégraux mais de drapeaux intégraux (suites croissantes d'éléments intégraux) au fin de donner une variété représentative pour l'ensemble de tous les drapeaux intégraux de $\sum$. Cette variété est étudiée par strates: le cas générique correspond aux drapeaux ordinaires tandis que les strates maigres sont constitués par les drapeaux singuliers.

Plus précisément: envisageons un système différentiel extérieur, qui, dans un repère fixé, s' écrit

$$
\Sigma \equiv \begin{cases}f_{\alpha}\left(x^{1}, \ldots, x^{n}\right)=0 & \left(\alpha=1, \ldots, r_{0}\right) \\ \theta_{\alpha} \equiv A_{\alpha i_{1}}\left(x^{1}, \ldots, x^{n}\right) d x^{i_{1}}=0 & \left(\alpha=1, \ldots, r_{1}\right) \\ \phi_{\alpha} \equiv A_{\alpha i_{1} i_{2}}\left(x^{1}, \ldots, x^{n}\right) d x^{i_{1}} \wedge d x^{i_{2}}=0 & \left(\alpha=1, \ldots, r_{2}\right) \\ \ldots \ldots & \end{cases}
$$

Les drapeaux intégraux peuvent être obtenus par récurrence en régardant successivement les équations de degré plus grand.

Les seules $f_{\alpha}=0$ définissent, en $\mathbb{R}^{n}$, un ensemble analytique que nous appelons $Z$;

pour chacun de ses points réguliers, soit $x$, on définit le système linéaire homogène en les inconnues $u \Sigma_{0}(x) \equiv A_{\alpha i}(x) u^{i}=0,\left(\alpha=1, \ldots, r_{1}\right)$;

pour chaque solution $u_{1}$ de $\Sigma_{0}(x)$ (et donc pour chaque drapeau intégral $\delta \equiv\{x\} \subset$ $\left.\left(x ; u_{1}\right)\right)$, on définit le système $\Sigma_{1}\left(x ; u_{1}\right)$ polaire de $u_{1}$, c'est à dire le système linéaire 
homogéne en les $u$

$$
\Sigma_{1}\left(x ; u_{1}\right) \equiv \begin{cases}A_{\alpha j}(x) u^{j}=0 & \left(\alpha=1, \ldots, r_{1}\right), \\ A_{\alpha i j}(x) u_{1}^{i} u^{j}=0 & \left(\alpha=1, \ldots, r_{2}\right)\end{cases}
$$

chaque solution $u_{2}$ orthogonale a $u_{1}$ de ce système nous donne un 2-drapeau intégral $\delta_{2} \equiv\{x\} \subset\left(x ; u_{1}\right) \subset\left(x ; u_{1}, u_{2}\right)$ et aussi un système $\Sigma_{2}\left(x, u_{1}, u_{2}\right)$; etc.

Les indices de Cartan sont alors les plus grandes valeurs possibles des rangs de ces systèmes polaires, c'est à dire $s_{i}=\max$ rang $\Sigma_{i}$ et le genre est l'entier $p$ tel que $n-p=$ $s_{0}+\ldots+s_{p}$. On a la

Proposition 2 (v. [1]). Le système $\Sigma$ détermine une suite $\left\{\mathcal{B}_{k}\right\}(k=1, \ldots, p)$ d'espaces fibrés; base de $\mathcal{B}_{k}$ est $W_{k-1}$, ouvert dense dans $\mathcal{B}_{k-1}$ (pour $k \geq 2 ; W_{0}$ est ouvert dense dans $Z$ ), et la fibre est $L_{k}:=\mathbb{P}^{n-s_{0}-\ldots-s_{k-1}-k}$. Un élément intégral de $\sum$ est ordinaire si et seulement si il est extrême d'un drapeau de $\mathcal{B}_{k}$.

Un apparent désaccord entre la dimension de $\mathcal{B}_{k}$, que l'on calcule immédiatement, et le nombre des paramètres desquelles depend la solution général de $\sum$ est expliqué en [3].

Cette manière de traiter la question porte à une généralization: en introduisant un multi-indice $\tau=\left(t_{h}, \ldots, t_{k-1}\right) \in \mathbb{N}^{k-h}$ où les $t_{i}$ généralisent les indices de Cartan $s_{i}$ (étant definis comme les rangs, non plus nécessairement maxima, des systèmes polaires successifs de $\sum$ ), nous pouvons étudier (et construire effectivement d'une façon analogue à $\mathcal{B}_{k}$ ) les variétés ${ }_{h} \mathcal{C}_{k}^{\tau}$ représentatives des drapeaux intégraux, de dimension $k$, qui sont ordinaires entre la dimension 0 et la dimension $h(h<k)$, singuliers à partir de la dimension $h+1$; la singularité s'exprime à l'aide des indices $t_{i}$. Il y a donc la

Proposition 3 (v. [4]). Au système différentiel extérieur $\sum$ se trouve associée la famille $\left\{{ }_{h} \mathcal{C}_{k}^{\tau}\right\}$ d'espaces fibrés (éventuellement triviaux), où $0 \leq h \leq p-2, k>h, 0 \leq$ $t_{h} \leq s_{h}-1, t_{h}+\ldots+t_{k-1} \leq s_{h}+\ldots+s_{k-1}$. Base de ${ }_{h} \mathcal{C}_{k}^{\tau}={ }_{h} \mathcal{C}_{k}^{t_{h}, \ldots, t_{k-1}}$ est ${ }_{h} \Phi_{k-1}^{\tau}$, ouvert dense dans un convenable sous-ensemble de ${ }_{h} \mathcal{C}_{k-1}^{\tau}$, (c'est à dire de ${ }_{h} \mathcal{C}_{k-1}^{\tau}$ duquel on a enlevé les sous-ensambles corréspondants aux valeurs $\left.t>t_{k-1}\right)$; la fibre est donnée par l'espace projectif $\mathbb{P}^{n-s_{0}-\ldots-s_{h-1}-t_{h}-\ldots-t_{k-1}-k}$.

(Avec cette notation nous pouvons écrire $\mathcal{B}_{k}$ même comme ${ }_{k} \mathcal{C}_{k}$ ).

3. Nous pouvons alors rassembler les raisonnements de 1) ed de 2).

Bornons nous aux systèmes différentiels extérieurs $\sum$ en $\mathrm{d} x^{1}, \ldots, \mathrm{d} x^{n}$ qui, en un repère fixé une fois pour toutes, ont coefficients constants. L'ensemble de ces systèmes si leurs idéaux parcourent $\mathcal{H}^{\rho}\left(l_{1}, \ldots, l_{q} ; n\right)$, peut se voir comme un (unique) système $\Sigma\left(l_{1}, \ldots, l_{q} ; \rho\right)$ à coefficients indéterminés.

Indiquons avec $s_{0}, \ldots, s_{p}$ les indices de Cartan de $\Sigma\left(l_{1}, \ldots, l_{q} ; \rho\right)$, avec $t_{0}, \ldots, t_{h}$ les nouveaux indices de 2 ) et considérons les variétés des k-drapeaux intégraux, ordinaires et caractéristiques du système à coefficients indéterminés (de sort que, si l'on spécialise les variables qui y figurent aux valeurs définissant un certain système $\sum$ fixe, on retrouve les fibrés $\mathcal{B}_{k}$ et ${ }_{h} \mathcal{C}_{k}^{\tau}$ que nous venons d'introduire).

Pour $h, k \in \mathbb{N}$ avec $h \leq k$, et $\tau:=\left(t_{h}, \ldots, t_{k-1}\right) \in \mathbb{N}^{k-h}\left(\operatorname{avec} t_{h}<s_{h}\right.$ et $t_{h}+\ldots+$ $\left.t_{k-1} \leq s_{h}+\ldots+s_{k-1}\right)$, définissons ${ }_{h}^{\rho} \mathcal{C}_{k}^{\tau}\left(l_{1}, \ldots, l_{q}\right)$ la variété représentative des k-drapeaux 
intégraux de $\Sigma\left(l_{1}, \ldots, l_{q} ; \rho\right)$ ordinaires entre les dimensions 1 et $h$ et singuliers entre $h$ et $k$. Comme nous avons vu, la singularité s'exprime au moyen des indices $t_{h}, \ldots, t_{k-1}$.

Pour $k=h$ on peut retrouver en ce définition aussi la variété ${ }^{\rho} \mathcal{B}_{k}\left(l_{1}, \ldots, l_{q}\right):=$ ${ }_{k}^{\rho} \mathcal{C}_{k}\left(l_{1}, \ldots, l_{q}\right)$ des $k$-drapeaux ordinaires. Nous avons donc la

Proposition 4. ${ }_{h}^{\rho} \mathcal{C}_{k}^{t_{h}, \ldots, t_{k-1}}\left(l_{1}, \ldots, l_{q}\right)$ est un fibré, ayant pour base un sousensemble de ${ }_{h}^{\rho} \mathcal{C}_{k-1}^{t_{h}, \ldots, t_{k-2}}\left(l_{1}, \ldots, l_{q}\right)$ et pour fibre l'espace projéctif $\mathbb{P}^{n-s_{0}-\ldots-s_{h-1}-t_{h}-\ldots-t_{k-1}-k}$.

De la même façon que pour un système fixe $\sum$ la réunion des fibrés $\mathcal{B}_{k}$ et des ${ }_{h} \mathcal{C}_{k}^{\tau}$, lorsque $h, k, \tau$ parcourent leurs ensembles, constitue la variété de tous les drapeaux intégraux de $\sum$, aussi pour $\Sigma\left(l_{1}, \ldots, l_{q} ; \rho\right)$ (donc: avec $\rho$ fixé) la réunion $\operatorname{des}{ }_{h}^{\rho} \mathcal{C}_{k}^{\tau}\left(l_{1}, \ldots, l_{q}\right)$ au varier de $h, k, \tau$ nous donne tous les drapeaux intégraux de $\Sigma\left(l_{1}, \ldots, l_{q} ; \rho\right)$.

Nous avons donc une description complète obtenue par strates de la variété représentative de tous ces drapeaux et encore les drapeaux ordinaires en constituent le cas générique et les drapeaux caractéristiques les strates maigres.

Si en plus nous faisons varier le multi-indice $\rho$, nous obténons alors l'ensemble de tous les drapeaux intégraux de tous les systèmes différentiels extérieurs à coefficients indterminés dont les idéaux détérminent les caractères $\left\{l_{1}, \ldots, l_{q}, m_{1}, \ldots, m_{q}\right\}$. Nous avons ainsi une double stratification.

4. Une description géometrique pareille à celle montrée en A pour les idéaux de formes extérieures réelles (et donc pour les systèmes différentiels extérieurs à coefficients constants) est possible aussi pour l'ensemble des systèmes différentiels extérieurs qui, en un repère fixé, ont pour coefficients des polynômes homogènes qui pour chaque équation ont tous le même degré variable avec l'équation.

En [6] nous avons étudié cet ensemble en introduisant des caractères convenables et une convenable relation d'equivalence et nous avons ainsi étudié les variétés $S\left(l_{1}, \ldots, l_{q} ; \lambda ; n\right)$ représentatives pour les systèmes de ce type.

Le problème se pose de décrire les variétés des drapeaux intégraux lorsque les systèmes différentiels extérieurs sont indéterminés et parcourent $S\left(l_{1}, \ldots, l_{q} ; \lambda ; n\right)$.

\section{Références}

[1-2] B. Bigolin et C. Perelli Cippo, Drapeaux intégraux ordinaires d'un système différentiel extérieur I-II, Bull. Soc. Sci. Lettres Łódź 39, no. 8-9 (Sér. Rech. Déform. 7, no. 62-63) (1989), 17 pp. + 17 pp.

[3] C. Perelli Cippo, Condizioni di Schubert per gli elementi integrali di un sistema differenziale esterno, Rend. Ist. Lombardo 122 (1988).

[4] B. Bigolin et C. Perelli Cippo, Remarques sur les drapeaux intégraux des systèmes différentiels extérieurs (drapeaux caractéristiques), Bull. Soc. Sci. Lettres Łódź 41, no. 10 (Sér. Rech. Déform. 10, no. 100) (1991), 81-98.

[5] C. Perelli Cippo et S. Vassallo, Ideali non omogenei di polinomi: i fibrati $B$ e $C$, Rend. Ist. Lombardo 125 (1991).

[6] C. Perelli Cippo et S. Vassallo, Sistemi differenziali esterni a coefficienti polinomi omogenei, Preprint: Quaderni del Seminario Matematico di Brescia, no. 6/92. 